\section{Frontal Behavioral Inventory}

Chava Creque $^{1}$ and Stephanie A. KolakowskyHayner $^{2}$

${ }^{1}$ Department of Psychology and Neuroscience, University of Colorado Boulder, Boulder, CO, USA

${ }^{2}$ Icahn School of Medicine at Mount Sinai, New York, NY, USA

\section{Synonyms}

FBI

\section{Definition}

The Frontal Behavioral Inventory (FBI) is a 24item questionnaire that measures behaviors such as apathy, indifference, disorganization, inattention, personal neglect, aspontaneity, inflexibility, concreteness, loss of insight, logopenia, verbal apraxia, and alien hand. The test is often used to assess frontal lobe dementia. Minimal formal training is required to administer the questionnaire, which takes approximately $15-30 \mathrm{~min}$ to complete. The FBI is typically administered as an interview to caregivers to assess personality or behavioral changes from the patient's premorbid abilities. A four-point scale (none, mild, moderate, and severe) for each item is used and is dependent on the caregiver's response. The FBI is intended to serve as a quantitative measure to determine the severity of impairment and to possibly assess a change due to therapeutic intervention.

\section{Cross-References}

Frontal Lobe

- Frontal Lobe Syndrome

\section{References and Readings}

Gordon, P., Yuanjia, W., Doorish, C., Lewis, M., Battista, V., Mitsumoto, H., et al. (2007). A screening assessment of cognitive impairment in patients with ALS. Amyotrophic Lateral Sclerosis, 8(6), 362-365.

Gorenstein, E. (1982). Frontal lobe functions in psychopaths. Journal of Abnormal Psychology, 91(5), 368-379.

Kertesz, A., Nadkarni, N., Davidson, W., \& Thomas, A. W. (2000). The frontal behavioral inventory in the differential diagnosis of frontotemporal dementia. Journal of the International Neuropsychological Society, 6(04), 460-468.

Milan, G., Lamenza, F., Iavarone, A., Galeone, F., Lorè, E., De Falco, C., et al. (2008). Frontal behavioural inventory in the differential diagnosis of dementia. Acta Neurologica Scandinavica, 117(4), 260-265. 